\title{
Ungewöhnlicher Fall eines schweren Polytraumas beim Kleinkind - Happy End trotz Fallstricken
}

Andreas Klinger, Patrick Sperling, Hendrik Jansen, Johannes Wirbelauer, Daniel Vergho, Florian Seitz, Norbert Roewer,

Thomas Meyer, Ralf Michael Muellenbach

\section{Der Fall}

\section{Anamnese und Befund}

Der Rettungsdienst wird zu einem Verkehrsunfall alarmiert. Ein 16 Monate alter Junge (ca. 12 kgKG) wurde im Kinderbuggy sitzend von einem Geländewagen auf dem Gehweg erfasst und gegen eine Betonmauer gequetscht.

Der 3 Minuten später eintreffende Notarzt findet folgendes Szenario vor: Der Kinderbuggy ist stark verformt, das Kleinkind jedoch frei zugänglich und schreit (GCS Augen/ Sprache/Motorik 2/4/6=11). Der initiale Trauma-Check ergibt den Verdacht auf Frakturen beider Oberschenkel, eine Instabilität des Beckens sowie - unter blutgetränkter Kleidung - eine ca. $3 \mathrm{~cm}$ große, stark blutende Wunde knapp oberhalb der Symphyse. Weitere Verletzungen können nicht festgestellt werden. Die Rekapillarisierungszeit ist noch knapp <2 s und der Brachialispuls tastbar.

Der Notarzt stellt folgende Verdachtsdiagnosen:

- V.a. offenes Beckentrauma

- V.a. Femurfrakturen beidseits

- hämorrhagischer Schock (präklinisch nicht behebbares C-Problem)

\section{Präklinische Versorgung und Transport}

Der Notarzt legt einen i. o. Zugang in der linken Tibia. Über diesen werden eine Analgosedierung mit $50 \mu \mathrm{g}$ Fentanyl i.o. plus $5 \mathrm{mg}$ Diazepam rektal appliziert sowie $200 \mathrm{ml}$ kristalloides Volumen substitutiert. 19 Minuten nach primärer Alarmierung wird ein 2. Notarzt nachgefordert mit der Bitte zur fachlichen Unterstützung und Beratung der weiteren Einsatztaktik („stay and play“ vs. „load-go-and-treat“) sowie der erforderlichen Maßnahmen. Dieser stellt bei seiner Traumauntersuchung im Rettungswagen keine neuen Verletzungen fest. Als Vitalzeichen zeigen sich:

- Herzfrequenz 130/min

- Brachialispuls tastbar

- $\mathrm{SpO}_{2} 99 \%$

- Rekapillarisierungszeit verlängert (ca. 3 s)

Bei suffizienter Spontanatmung und einem GCS von 11 wird hochdosiert Sauerstoff über eine Inhalationsmaske verabreicht. Das Becken wird mittels Vakuummatratze stabilisiert, die stark blutende Wunde im Bereich der Symphyse zur äußerlichen Blutungskontrolle manuell komprimiert, wobei klinisch die Schockzeichen (s. o.) zunehmen.

Da sich zum einen der klinische Zustand des Kindes rapide verschlechtert und zum anderen ein überregionales Traumazentrum nur 7 min entfernt liegt, wird zugunsten des zügigen Transports auf weitere medizinische Maßnahmen wie Intubation, Anlage eines i.v. oder eines weiteren i.o. Zugangs verzichtet (Transportbeginn 3 min nach Eintreffen des 2. Notarztes bzw. 25 min nach Alarmierung). Die Einsatztaktik wird somit von „stay-andplay“ auf „load-go-and-treat“ geändert.

Die Übergabe an das telefonisch vorverständigte Schockraumteam (Arzt-Arzt-Gespräch) erfolgt ca. 32 min nach primärer Alarmierung.

\section{Innerklinische Versorgung}

Bei Ankunft im Schockraum ist der Kreislauf trotz präklinischer kristalloider Volumensubstitution (ca. $500 \mathrm{ml}$ ) instabil. Die Extremitäten sind kalt und zentralisiert und die Rekapillarisierungszeit ist deutlich verlängert. Die HbKonzentration beträgt $8,5 \mathrm{~g} / \mathrm{dl}$ und bei einer metabolischen Azidose mit einem $\mathrm{pH}$ von 7,23 und einem BE von $-10 \mathrm{mmol} / \mathrm{l}$ liegt ein schwerer hämorrhagischer Schock vor. Bei einem geschätzten Blutverlust von ca. 200-300 ml (Verlust von ca. 30-40\% des Blutvolumens bei einem geschätzten Körpergewicht von $12 \mathrm{~kg}$ ) werden umgehend über einen neu etablierten i.v. Zugang (20 G periphere Venenverweilkanüle) in der linken V. jugularis externa 2 ungekreuzte Erythrozytenkonzentrate der Blutgruppe 0 negativ transfundiert. Der Patient wird zügig intubiert und beatmet.

In der kurzgefassten Schockraumdiagnostik (klinische Untersuchung, Abdomensonografie, Röntgen: Thorax u. Becken) zeigt sich bei skrotalem Décollement die linke untere Extremität insuffizient durchblutet. Außerdem findet sich eine von der Inguinalfalte links bis nach perineal ziehende, tief klaffende, ca. $10 \mathrm{~cm}$ messende Wunde, aus der sich bei Sistieren der Kompression ein dicker venöser Blutstrom entleert. Außerdem wird eine instabile, stark nach dorsal und distal dislozierte Becken-C-Verletzung festgestellt: beidseits anteriore Beckenringfraktur, Fraktur beider Schambeinäste und Sprengung des linken Ileosakralgelenks. 
Bei äußerer Blutungskontrolle durch manuelle Kompression und befürchteter weiterer Dislokation der Fraktur wird auf die Anlage einer Beckenschlinge verzichtet und die zügige operative Intervention angestrebt. Zur Kreislauftherapie wird ultraschallgestützt ein ZVK in die V. jugularis interna rechts eingelegt. Bei traumatisch induzierter Koagulopathie sind im Weiteren eine Massivtransfusion (1200 ml EK, $400 \mathrm{ml} \mathrm{FFP} \mathrm{und} 150 \mathrm{ml}$ TK) und die Substitution von Gerinnungsfaktoren ( $2 \mathrm{~g}$ Fibrinogen, 250 IE Antithrombin III, 350 IE PPSB) notwendig.

Im Rahmen der Notfalllaparotomie zeigen sich neben der oben beschriebenen Beckenringfraktur die Durchtrennung der V. und A. iliaca externa und des Plexus lumbosacralis links sowie eine komplette Urethraruptur mit konsekutiver Verlagerung der Harnblase nach kranial. Nach Blutstillung und Rekonstruktion der lliakalgefäße erfolgt die Osteosynthese des Beckens mittels Platten und Fixateur externe sowie die Rekonstruktion von Blase, Hoden und Urethra.

Am 11. postoperativen Tag wird der Patient extubiert, am 24. Tag auf Normalstation verlegt und nach multiplen Revisionseingriffen am 66. Tag in gutem Allgemeinzustand in die Rehabilitation entlassen. Bei Plegie des linken Beins steht allerdings die Rekonstruktion des Plexus lumbosacralis links noch aus.

\section{Diskussion}

Pädiatrische Notfälle sind im Notarztdienst mit ca. 3,5$5,5 \%$ sehr selten [1]. Die Versorgung eines schwer polytraumatisierten Säuglings oder Kleinkindes ist dabei eine Rarität (ca. 1\% der polytraumatisierten Patienten) [2]. Im Gegensatz zu Kopfverletzungen (71\%) machen Verletzungen des Beckens bei polytraumatisierten Kindern nur 10\% der Fälle aus, wobei durch die Begleitverletzungen eine deutlich gesteigerte Letalität und Morbidität besteht [3].

\section{Präklinische Rettungszeit}

Aufgrund der geringeren physiologischen Reserven muss beim pädiatrischen Patienten generell - aber insbesondere beim hämodynamisch instabilen Kind - schneller als beim Erwachsenen entschieden werden, ob es sich um eine „load-go-and-treat“-Situation handelt. In dem geschilderten Fall war die gesamte präklinische Rettungszeit mit 32 Minuten innerhalb der „Golden Hour of Shock" und im Vergleich zu in der Literatur beschriebenen Rettungszeiten kurz [4-6]. Dies ist vor allem auf das urbane Umfeld zurückzuführen. In einem ruralen Umfeld kann die „Golden Hour of Shock“ nur durch frühzeitiges Einbeziehen der Luftrettung erreicht werden.

\section{Verzögerung durch späte Nachalarmierung des}

\section{Notarztes}

Bei detaillierter Analyse des Einsatzverlaufs fällt jedoch auf, dass vom Eintreffen des 1. Notarztes bis zur Nachforderung des 2. Notarztes nach 19 Minuten bzw. dessen Ein- treffen nach 22 Minuten viel Zeit verging. Die berechtigte Nachforderung weiterer Einsatzkräfte (2. Notarzt) wäre unmittelbar nach Erkennen der Verletzungsschwere bzw. der Notwendigkeit weiterer Unterstützung günstiger gewesen. Durch die rasche Nachforderung kann das Team früher optimal unterstützt werden, während eine verzögerte Nachforderung zu einer Gefährdung des Patienten führen kann. Alternativ hätte man auch den schnellen Transport in das Traumazentrum erwägen können.

\section{„Stay-and-play“ versus „load-go-and-treat“}

Das Notärzteteam entschied sich unter strenger NutzenRisiko-Evaluation am Unfallort, auf eine Narkoseinduktion und weitere Maßnahmen im Sinne einer „load-go-andtreat“-Strategie zu verzichten und den Transport 3 Minuten nach Eintreffen des 2. Notarztes (25 min nach dem Unfall) einzuleiten. Diese Entscheidung war auch retrospektiv sinnvoll, denn das präklinisch nicht beherrschbare C-Problem kann lediglich durch die schnellstmögliche operative Intervention therapiert werden. Eine präklinische Narkoseeinleitung hätte zweifellos zu einer weiteren Verzögerung geführt [7]. Somit wäre dieses Vorgehen auch im ruralen Umfeld sinnvoll.

\section{Analgosedierung}

Die im Rahmen des „load-go-and-treat“ durchgeführte suffiziente Analgosedierung wurde mit rektal appliziertem Diazepam und nach Anlage des i.o. Zugangs mit einer hohen Dosis Fentanyl $(50 \mu \mathrm{g}=4,12 \mu / \mathrm{kgKG})$ erreicht. Die rektale Medikamentenapplikation wurde als Erstmaßnahme bei unzureichenden alternativen Möglichkeiten gewählt. Falls verfügbar ist die Applikation mittels eines nasalen Zerstäubers aufgrund des schnelleren Wirkeintritts eleganter. Weiterhin ist bei einem Beckentrauma die Verletzung der Beckenorgane möglich, sodass die nasale oder die im Folgenden besprochene parenterale Applikation (i.v. und i.o.) günstiger und sicherer scheint [8].

Als Alternative zu einer Analgosedierung mit einem Opiat hätte Ketamin den Vorteil, weniger kreislaufdepressiv zu sein [8]. Zur Ermittlung der optimalen Medikamentendosierung können Merkhilfen wie „Notfallbänder“ hilfreich sein [9].

\section{Intraossärer Zugang und Punktionsort}

Im Säuglings- und Kleinkindalter ist bei zeitkritischen Notfällen und schwierigen Punktionsbedingungen der i. o. Zugang Mittel der ersten Wahl. Dieser lässt sich im Gegensatz zur i.v. Punktion schnell und sicher anlegen [10]. In unserem Fall wurde der i. o. Zugang in die linke Tibia angelegt. Liegt jedoch eine Beckenfraktur vor, sollte aufgrund des Risikos eines Paravasats und insuffizienter Medikamentenwirkung als alternativer Punktionsort z. B. der proximale Humerus gewählt werden [10]. Die Punktion größerer zentraler Gefäße wie der V. jugularis oder der V. femoralis (jedoch nicht bei Verletzung distal des 
Beckens) ist präklinisch in der Notfallsituation ohne Ultraschallkontrolle nahezu unmöglich [11], weswegen die korrekte Anlage eines i. o. Zugangs umso wichtiger ist.

\section{Blutstillung}

Das Stillen einer kritischen Blutung ist entscheidend für die Kreislaufstabilisierung. Im vorliegenden Fall kam es einerseits durch die offene, stark blutende Wunde und andererseits durch die instabile Beckenfraktur zu einer kritischen Blutung. Die Anlage eines Beckengürtels ist bei einer Beckenfraktur State of the Art [12]. Dieser ist im Gegensatz zu Säuglingen und Kleinkindern jedoch nur für den adulten Patienten kommerziell erhältlich. Daher wurde im vorliegenden Fall eine Vakuummatratze zur Stabilisierung der Beckenfraktur des Kleinkinds verwendet. Durch Anlage einer Tuchschlinge hätte möglicherweise die Kompression der Beckenfraktur verbessert werden können. Allerdings wäre dann die Kompression der offenen Wunde am Becken nicht mehr möglich gewesen.

\section{Voranmeldung mittels Arzt-Arzt-Gespräch}

Da ein polytraumatisiertes Kind aufgrund der Seltenheit auch für das innerklinische Team eine Besonderheit darstellt und die Transportzeit von 7 Minuten sehr kurz war, wirkte sich das direkte Arzt-Arzt-Gespräch zur Voranmeldung begünstigend auf die innerklinische Versorgungsqualität aus. Bei Voranmeldung ohne direkten Arzt-ArztKontakt kann es nach dem Phänomen der „stillen Post“ zu signifikanten Informationsverlusten bzw. -verfälschungen kommen [13]. Im geschilderten Fall konnte sich das innerklinische Team optimal auf das polytraumatisierte Kind einstellen und die notwendigen Medikamente samt Dosierungen, Zugängen, Kathetern und Tuben sowie die Notfalltransfusion vorbereiten und somit das gute Outcome erreichen.

\section{KERNAUSSAGEN}

- Das Hinzuziehen eines weiteren Notarztes ohne zeitliche Verzögerung kann vor allem bei seltenen bzw. akut vitalbedrohlichen Notfällen zur Patientensicherheit und Verbesserung der Versorgungsqualität beitragen. Die zusätzlichen Kräfte sollten aber zum frühestmöglichen Zeitpunkt angefordert werden.

- Beim nicht kontrollierbaren C-Problem hat der schnellstmögliche Transport in die nächstgelegene geeignete Klinik oberste Priorität. Daher sollten nur absolut notwendige lebensrettende Maßnahmen an der Unfallstelle erfolgen.

- Beim polytraumatisierten Kind ist eine Voranmeldung mittels Arzt-Arzt-Gespräch sehr wichtig.

- Die Anlage eines i. o. Zugangs ist lebensrettend. Als Punktionsorte kommen in allen Altersklassen die Tibia, der Femur sowie der Humerus infrage.
Interessenkonflikt

Die Autoren geben an, dass keine Interessenkonflikte vorliegen.

\section{Autorinnen/Autoren}

\section{Andreas Klinger}

Dr. med. Promotion 2011. Seit 2010 Assistenzarzt und Facharzt für Anästhesiologie (2017) am Universitätsklinikum Würzburg. Seit 2012 regelmäßige Tätigkeit als Notarzt im bodengebundenen Rettungsdienst. Seit 2014 Sprecher für den Verlegungsarzt- und Notarztdienst der Sektion Notfall- und Katastrophenmedizin des Universitätsklinikums Würzburg. Seit 2017 Instruktor für „Emergency Pediatric Care“. Provider ITLS, ACLS, EPC und Qualifikation „Leitender Notarzt“.

\section{Patrick Sperling}

Dr. med. Funktionsoberarzt der Abteilung für Kinderchirurgie an der Klinik und Poliklinik für Allgemein- und Viszeralchirurgie, Gefäß- und Kinderchirurgie des Universitätsklinikums Würzburg. Seit Januar 2018 Oberarzt der Klinik für Kinderchirurgie und Kinderorthopädie, Barmherzige Brüder Klinik St. Hedwig, Regensburg.

\section{Hendrik Jansen}

PD Dr. med. Leitender Oberarzt der Klinik und Poliklinik für Unfall-, Hand-, Plastische- und Wiederherstellungschirurgie des Universitätsklinikums Würzburg.

\section{Johannes Wirbelauer}

Prof. Dr. med. Leitender Oberarzt der Kinderklinik und Poliklinik des Universitätsklinikums Würzburg. Schwerpunkt: Pädiatrische Intensivmedizin.

\section{Daniel Vergho}

PD Dr. med. Oberarzt an der Klinik und Poliklinik für Urologie und Kinderurologie des Universitätsklinikums Würzburg. Seit Januar 2017 niedergelassener Urologe in Landau an der Isar.

\section{Florian Seitz}

Dr. med. Oberarzt der kinderchirurgischen Abteilung an der Klinik und Poliklinik für Allgemein- und Viszeralchirurgie, Gefäß- und Kinderchirurgie des Universitätsklinikums Würzburg.

\begin{abstract}
Norbert Roewer
Univ.-Prof. Dr. Dr. h. c. Direktor und Ordinarius an der Klinik und Poliklinik für Anästhesiologie des Universitätsklinikums Würzburg und stellvertretender ärztlicher Direktor des Universitätsklinikums Würzburg. Prof. Roewer gehört zum Herausgeberteam der AINS.
\end{abstract}

\section{Thomas Meyer}

Prof. Dr. med. Leiter der kinderchirurgischen Abteilung an der Klinik und Poliklinik für Allgemein- und Viszeralchirurgie, Gefäß- und Kinderchirurgie des Universitätsklinikums Würzburg. 


\section{Ralf Michael Muellenbach}

Prof. Dr. med. Direktor der Klinik für Anästhesiologie, Intensivmedizin, Notfallmedizin und Schmerztherapie, Klinikum Kassel. Promotion 2004, Habilitation 2009, 2012-2016 Leiter der anästhesiologischen Intensivstation der Klinik und Poliklinik für Anästhesiologie des Universitätsklinikums Würzburg (2014-2016 als geschäftsführender Oberarzt). Langjährige Erfahrung im boden- und luftgebundenen Rettungsdienst.

\section{Korrespondenzadresse}

\section{Dr. med. Andreas Klinger}

Klinik und Poliklinik für Anästhesiologie

Universitätsklinikum Würzburg

Oberdürrbacherstraße 6

97080 Würzburg

Klinger_a@ukw.de

\section{Literatur}

[1] Messelken M, Kehrberger E, Dirks B et al. The quality of emergency medical care in Baden-Wurttemberg (Germany): four years in focus. Dtsch Arztebl Int 2010; 107: 523-530

[2] Debus F, Lefering R, Frink $M$ et al. Das Polytrauma von Kindern und Jugendlichen - Auswahl der primärversorgenden Klinik und Bedeutung von kindertraumatologischen Kompetenzzentren. Unfallchirurg 2015; 118: 432-438

[3] Schneidmueller D, Wutzler S, Kelm A et al. Beckenverletzungen im Kindes- und Jugendalter - Eine retrospektive Analyse eines überregionalen Traumazentrums über 5 Jahre. Unfallchirurg 2011; 114: 510-516

[4] Westhoff J, Kroener C, Meller R et al. Eingeklemmte Fahrzeuginsassen in der Luftrettung - Einsatztaktik, rettungstechnische und notfallmedizinische Analyse am Beispiel einer Rettungshubschrauberstation. Unfallchirurg 2008; 111: 155-161

[5] Sefrin P, Distler K. Stellenwert der Zugangszeit in der Rettungskette. Anasthesiol Intensivmed Notfallmed Schmerzther 2001; 36: 742-748
[6] Bernhard M, Helm M, Lechleuthner A. Erstversorgung vor Ort oder schnellstmöglicher Transportbeginn? Notfall + Rettungsmedizin 2017; 20: 579-585

[7] Chaudery M, Clark J, Wilson MH et al. Traumatic intra-abdominal hemorrhage control: Has current technology tipped the balance toward a role for prehospital intervention? J Trauma Acute Care 2015; 78: 153-163

[8] Hoffmann F, Deanovic D. Präklinische Schmerztherapie bei Kindern und Jugendlichen. Notfall + Rettungsmedizin 2011; 14: $549-553$

[9] Heimberg E, Heinzel O, Hoffmann F. Typische Probleme bei Kindernotfällen - Mögliche Lösungsstrategien. Medizinische Klinik - Intensivmedizin und Notfallmedizin 2015; 110: $354-$ 359

[10] Helm M, Haunstein B, Schlechtriemen T et al. EZ-IO( $\left.{ }^{\circledR}\right)$ intraosseous device implementation in German Helicopter Emergency Medical Service. Resuscitation 2015; 88: 43-47

[11] Strauss JM, Denk A. Kinderanästhesie - Der schwierige Venenzugang. Anasthesiol Intensivmed Notfallmed Schmerzther 2013; 48: 258-263

[12] Deutsche Gesellschaft für Unfallchirurgie (federführend). S3Leitlinie Polytrauma/Schwerverletzten-Behandlung. AWMF Register-Nr. 012/019; Stand: 07/2016. Im Internet: http:// www.awmf.org/leitlinien/detail/ll/012-019.html; Stand: 05.04.2017

[13] Vernon DD, Furnival RA, Hansen KW et al. Effect of a pediatric trauma response team on emergency department treatment time and mortality of pediatric trauma victims. Pediatrics 1999; 103: 20-24

\section{Bibliografie}

DOI https://doi.org/10.1055/s-0043-109008 Anästhesiol Intensivmed Notfallmed Schmerzther 2018; 53: 220-223 @ Georg Thieme Verlag KG Stuttgart · New York ISSN 0939-2661 